\title{
Gateway Channel Hopping to I mprove Transmission Efficiency in Long-range IoT Networks
}

\author{
Dae-Young Kim ${ }^{1}$ and Seokhoon $\mathrm{Kim}^{2 *}$ \\ ${ }^{1}$ School of IT Engineering, Daegu Catholic University \\ Gyeongsan 38430 - South Korea \\ [e-mail: kimdy81@cu.ac.kr] \\ ${ }^{2}$ Department of Computer Software Engineering, Soonchunhyang University \\ Asan 31538 - South Korea \\ [e-mail: seokhoon@sch.ac.kr] \\ *Corresponding author: Seokhoon Kim
}

Received October 1, 2018; accepted November 19, 2018; published March 312019

\begin{abstract}
Intelligent services have expanded as Internet of Things (IoT) technology has evolved and new requirements emerge to accommodate various services. One new requirement is transmitting data over long distances with low-power. Researchers have developed low power wide area (LPWA) network technology to satisfy the requirement; this can improve IoT network infrastructure and increase the range of services. However, network coverage expansion causes several problems. The traffic load is concentrated at a specific gateway, which causes network congestion and leads to decreased transmission efficiency. Therefore, the approach proposed in this paper attempts to recognize and then avoid congestion through gateway channel hopping. The LPWA network employs multiple channels, so wireless channel hopping is available in a gateway. Devices that are not delay sensitive wait for the gateway to reappear on their wireless channel; delay sensitive devices change the wireless channel along the hopping gateway. Thus, the traffic load and congestion in each wireless channel can be reduced improving transmission efficiency. The proposed approach's performance is evaluated by computer simulation and verified in terms of transmission efficiency.
\end{abstract}

Keywords: IoT, LPWA, channel hopping, transmission efficiency, gateway

A preliminary version of this paper was presented at APIC-IST 2018, and was selected by the conference review process. This research was supported by the MSIT(Ministry of Science, ICT),Korea, under the ITRC(Information Technology Research Center) support program (IITP-2018-2015-0-00403) supervised by the IITP(Institute for Information \&communications Technology Promotion), and this work was supported by the Soonchunhyang University Research Fund. 


\section{Introduction}

These days, small embedded systems called objects include communication and computing capabilities. All objects can be connected to a network to provide connectivity between objects, thus becoming the Internet of Things (IoT) [1-4]. The IoT provides various service opportunities. Information gathered from tiny devices can create more efficient intelligent services within industries and cities. Through context awareness created using such information, it is possible to provide a safe and convenient urban environment and improve factory productivity. IoT services limited to personal space are expanding to wider areas with the advent of Low Power Wide Area (LPWA) networks.

The LPWA network covers a wide area of up to $15 \mathrm{~km}$ and operates with very low-power when computing and communicating. It delivers data over a long distance by employing small size data and using a very low data rate. It transmits data over an unlicensed frequency band, each data packet is only a few hundred bytes, and the data rate is only tens of kbps. The application services of LPWA networks rely on simple message-based information gathering [5-10]. Therefore, long-range IoT services such as Smart City, Smart Factory, Smart Meter, Smart Military, and Smart Agriculture are suitable applications that use LPWA networks. There are several LPWA communication technologies; i.e., LoRaWAN [11], SigFox [12], and Weightless [13]. These technologies are de facto standards for LPWA communication. Among them, LoRaWAN is a representative communication technology; it is a star-topology network consisting of numerous end-devices, gateways, and a control server. End-devices are long-range IoT devices that transmit data over LoRa wireless. Gateways are a type of bridge that convert LoRaWAN messages into IP packets and transmit them; they connect to a control server through the IP network. The control server manages the LPWA network and gathers information from end-devices.

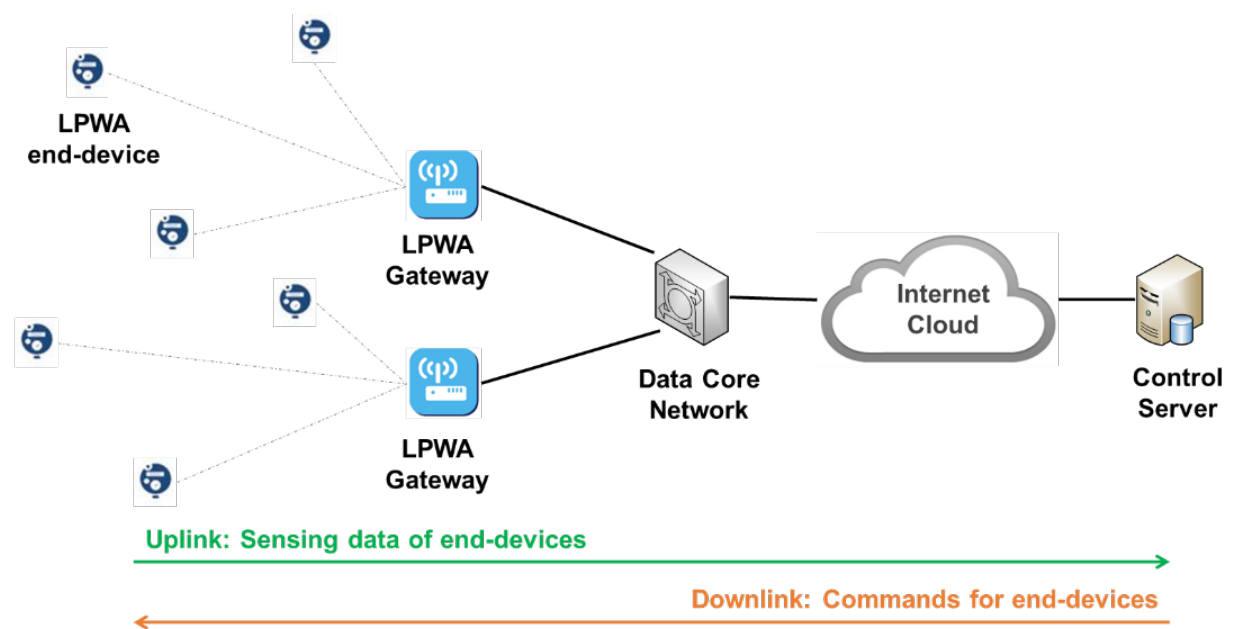

Fig. 1. LPWA network

In the LPWA network, uplink traffic is sensing information from end-devices and downlink traffic is commands for the behaviors of end-devices. In the IoT service domain, there are a lot of IoT devices (i.e., end-devices). In addition, because they have a long transmission range, a 
gateway can connect to more IoT devices [14-16]. Therefore, traffic load can be concentrated on a gateway, which can experience congestion as a bottleneck point when burst traffic is being generated. Fig. 1 shows the architecture of the LPWA network; congestion is a critical problem in LPWA networks. Mobile networks such as cellular adjust the amount of traffic during data transmission, while network protocols support avoiding congestion. However, because LPWA networks do not employ IP over wireless and have insufficient resources, they cannot apply the mobile network's congestion avoidance scheme. Fortunately, LPWA networks have multiple wireless channels and can change which wireless channel that use [14]. Therefore, this paper proposes a method to avoid congested wireless channels and improve the LPWA network's transmission efficiency.

In the proposed method, a gateway monitors traffic on the wireless channel in use and predicts wireless channel congestion through learning. If it predicts congestion, it moves to another wireless channel. It shares the wireless channel movement pattern when an end-device joins the LPWA network, which is determined by the control server. When a gateway moves the wireless channel, end-devices have to wait for the gateway to return to the current wireless channel. If an end-device has high priority, it moves wireless channel along with the moved gateway and can transmit data without waiting for the gateway to move back. After a certain period, end-devices move to another wireless channel or abandon data transmission. The wireless channel's congested state can be released.

The remainder of this paper is organized as follows. Section 2 describes related works and Section 3 presents the proposed congestion avoidance method. In Section 4, performance evaluation is carried out by computer simulation. Finally, Section 5 concludes this paper.

\section{Related Work}

The LPWA network uses a simple method for data transmission; it performs wireless channel sensing before transmitting data. After that, if a wireless channel is not busy, it sends a data frame. LPWA accesses the wireless channel by employing ALOHA [17] as a medium access control. ALOHA sends data to the wireless channel without any message exchange if there is data to send and performs random backoff if the data transmission fails. LPWA technology uses ALOHA because of its communication characteristics. As mentioned in the previous section, the system can transmit data frames far with very low power. For this wireless communication, LPWA uses a robust PHY modulation scheme for low receiver sensitivity; it transmits data frames at a very low data rate ( $50 \mathrm{kbps})$; therefore, LPWA experiences long transmission delays. ALOHA rarely uses control messages for data frame transmission, so LPWA can reduce transmission delays for message exchanges on transmissions.

Wireless networks commonly use the CSMA/CA [17] scheme as a medium access control. Network Allocation Vector (NAV) is set to avoid effective frame collisions during data transmission; it calculates the time a sending device occupies a wireless channel through the exchange of additional control messages, which leads to improved channel utilization in wireless networks. However, the exchange of control messages in the LPWA network is burdensome. Although long delay times are required for data transmission, the exchange of control messages may require longer time. Thus, most LPWA networks use an ALOHA-based simple transmission scheme.

The ALOHA and CSMA/CA approach random access to acquire wireless media means that the possibility of collision cannot be ruled out. Applying time division multiple access (TDMA) [17] to the LPWA network means that the transmission of a successful data frame 
without collision can be guaranteed. TDMA schedules data transmission and uses dedicated slots to guarntee transmission. There are numerous end-devices in an LPWA network; when applying TDMA, numerous end-devices should be reflected in the transmission schedule. In addition, the schedule cannot be changed easily if it has been completed; this means that the TDMA cannot provide scalability for the LPWA network.

As such, LPWA communication is a data transmission technology that has a large restriction to consider scalability, low-power, and long-distance transmission. It cannot use complicated control mechanisms. Nevertheless, there is a possibility of burst traffic transmissions by numerous end-devices, and this network congestion further degrades the transmission efficiency. Therefore, LPWA services require an effective way to avoid network congestion. Reducing network congestion requires reducing the amount of traffic that participates in the network; end-devices should minimize traffic incoming to the network. The proposed scheme avoids network congestion through the wireless channel hopping of a gateway. When the gateway moves over a wireless channel, the higher priority end-devices follow the gateway. The amount of traffic on the LPWA network reduces as the gateway hops through several wireless channels. Network congestion is then resolved when the gateway returns to its original wireless channel. Multiple wireless channels should be used when hopping wireless channels. Network congestion avoidance is possible through the proposed gateway channel hopping because LPWA technology is available in multiple wireless channels in the ISM frequency band.

Network congestion becomes predictable through learning; there have been several studies of network state prediction using learning algorithms in wireless networks performed through traffic monitoring. Whether the LPWA network is congested is determined through a classifier algorithm based on network data collected during traffic transmission. The gateway relays traffic between the IoT domain and a control server, so it can monitor traffic flows on the network and collect network data. Therefore, the gateway can predict the congestion state, which can change the wireless channel to avoid the congestion state.

Long-range IoT services employ many end-devices but cannot use complicated mechanisms to avoid the congestion state. The proposed method can avoid network congestion in a simple and effective manner, therefore, it is suitable for long-range IoT technology and applicable in various LPWA services.

\section{Gateway Channel Hopping}

\subsection{Network State Prediction}

LPWA communication conditions are poor. Many end-devices transmit data in the unlicensed frequency band And long delays occurs because device uses low power for long distances at low data rates. Therefore, anticipating and making preliminarily responses to network conditions is necessary. Supervised learning algorithms are widely used to predict the network state; they perform inference to predict the current given data from a training dataset. The proposed method uses a naïve Bayesian classifier algorithm for the prediction. A gateway constructs a training dataset with information on the signal strength and the transmission rate when receiving traffic. Several studies have exploited the information to identify the network state.

Naïve Bayesian classifier is based on the statistical Bayes rule and is used to classify binomial states; it classifies the current state by calculating the probabilities that can occur at the present using a dataset of past experiences [18-23]. The past dataset becomes the training 
data $(x)$ with which to predict the current state, and classification $(v)$ by the Bayes rule goes as follows:

$$
s=\arg _{y} P(y \mid x)=\arg _{y} \max _{y} \frac{P(x \mid y) P(y)}{P(x)},
$$

where $y$ is the past state. In the equation, $P(x)$ does not affect the state decision in Equation (1); therefore, it can be rewritten as

$$
s=\arg \max _{y} P(x \mid y) P(y) .
$$

The training data $x$ is divided into $x_{1}$ and $x_{2}$ depending on the information type such as the signal strength and transmission rate; $x_{1}$ and $x_{2}$ are conditionally independent for $y$, and Equation (2) can thus be represented as

$$
\begin{aligned}
s=\arg \max _{y} P(x \mid y) P(y) & =\arg \max _{y} P\left(x_{1} \mid y\right) P\left(x_{2} \mid y\right) P(y) \\
& =\arg \max _{y} \prod_{i=1}^{2} P\left(x_{i} \mid y\right) P(y)
\end{aligned}
$$

Probabilities are obtained in Equation (3) by the statistic of the monitoring traffic in the gateway. If $m$ training data in the statistic is given, the probabilities of $x$ and $y$ are calculated using the indicator function ${ }^{\dagger}$. The probability calculation relies on historical data (i.e., a priori statistics). Although historical data is important, a system should be able to calculate the appropriate probability even without historical data. Therefore, the probability calculation can be corrected through Laplace smoothing $[19,20]$ and the probabilities are represented as

$$
\begin{aligned}
& P\left(x_{i} \mid y=1\right)=\frac{\sum_{n=1}^{m} 1\left\{x_{i}^{n}=1, y^{n}=1\right\}+1}{\sum_{n=1}^{m} 1\left\{y^{n}=1\right\}+2} \\
& P(y=1)=\frac{\sum_{n=1}^{m} 1\left\{y^{n}=1\right\}+1}{m+2} \\
& P\left(x_{i} \mid y=0\right)=\frac{\sum_{n=1}^{m} 1\left\{x_{i}^{n}=1, y^{n}=0\right\}+1}{\sum_{n=1}^{m} 1\left\{y^{n}=0\right\}+2}, \\
& P(y=0)=\frac{\sum_{n=1}^{m} 1\left\{y^{n}=0\right\}+1}{m+2}
\end{aligned}
$$

where $\mathrm{y}=1$ means that congestion has occurred, and $\mathrm{y}=0$ means it has not. Laplace smoothing adds 1 to the numerator and 2 to the denominator ${ }^{\ddagger}$.

\subsection{Wireless Channel Hopping}

End-devices the select proper wireless channel to connect to the LPWA network. They join the

\footnotetext{
${ }^{\dagger}$ Indicator function $(1\{\cdot\})$ means it has a value of 1 if it belongs to a set and 0 if it does not.

‡ Congestion has binomial states and the number of states is added to the denominator.
} 
LPWA network by sending a Join Request Message to a network server, which allows them to join the network. The gateway creates a channel-hopping plan and shares it with the end-devices. The gateway changes its wireless channel when it predicts congestion; initially, it shares the plan for channel hopping when an end-device joins the LPWA network. The gateway updates the channel-hopping plan at every predetermined period and shares it with the end-devices in the network. Thus, the end-device that has urgent data can follow the gateway that moved wireless channel and can perform data transmission there. Fig. 2 shows the procedure by which the gateway shares its channel-hopping plan.

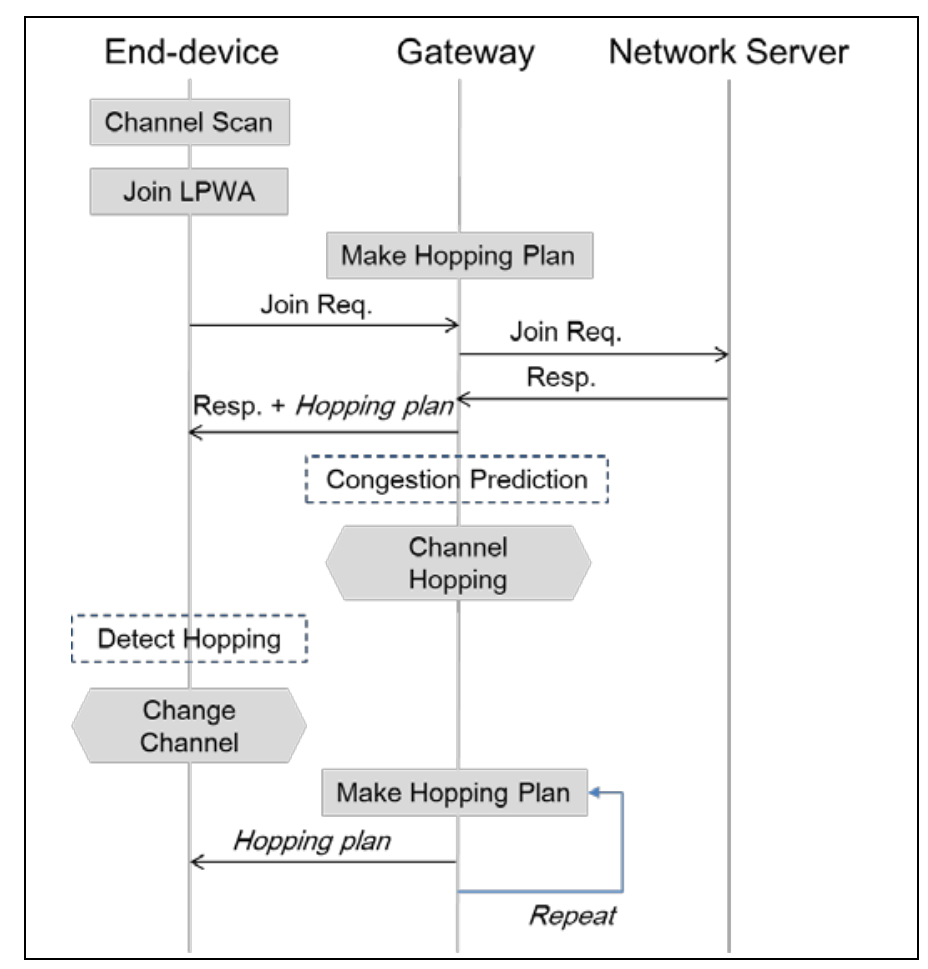

Fig. 2. The procedure by which the gateway shares its channel-hopping plan

After the gateway moves to another wireless channel, end-devices without urgent data stop transmitting data and wait for the gateway to return. When the gateway that moved wireless channel returns, it informs its end-devices through message broadcasting. Then, the end-devices reattempt to transmit their data. Fig. 3 shows end-device's operation after the gateway moves wireless channel. While the gateway has moved, the original wireless channel can avoid congestion, and several end-devices following the moved gateway means that congestion on the original wireless channel can be resolved when the original wireless channel is reactivated (i.e., the gateway returns the wireless channel).

The gateway sets a timer to return to its original wireless channel when it moves to another wireless channel. If it predicts that congestion will reoccur at the moved wireless channel, the gateway can move wireless channel again; however, when the timer to return expires, the gateway should return to its original wireless channel. The gateway schedules the channel-hopping plan and shares this with its end-devices; thus, the end-devices can follow the moved gateway if they have urgent data to send using the shared channel-hopping plan. Fig. 4 shows the gateway's operation during congestion; the gateway records its congestion history 
and reflects that history in the next hopping schedule. According to the congestion history, the gateway classifies available wireless channels into grades and randomly selects the moving channel sequences in the grades. The network server provides available channel information to the gateways.

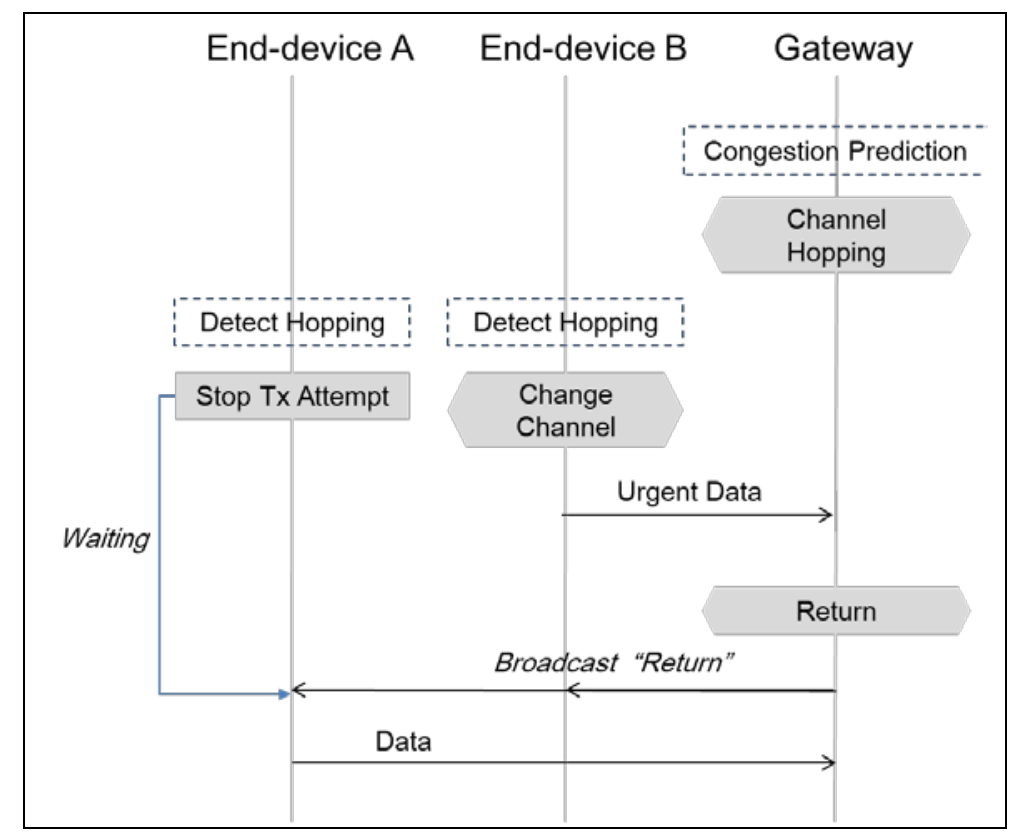

Fig. 3. The end-devices’ operation after the gateway has moved wireless channel

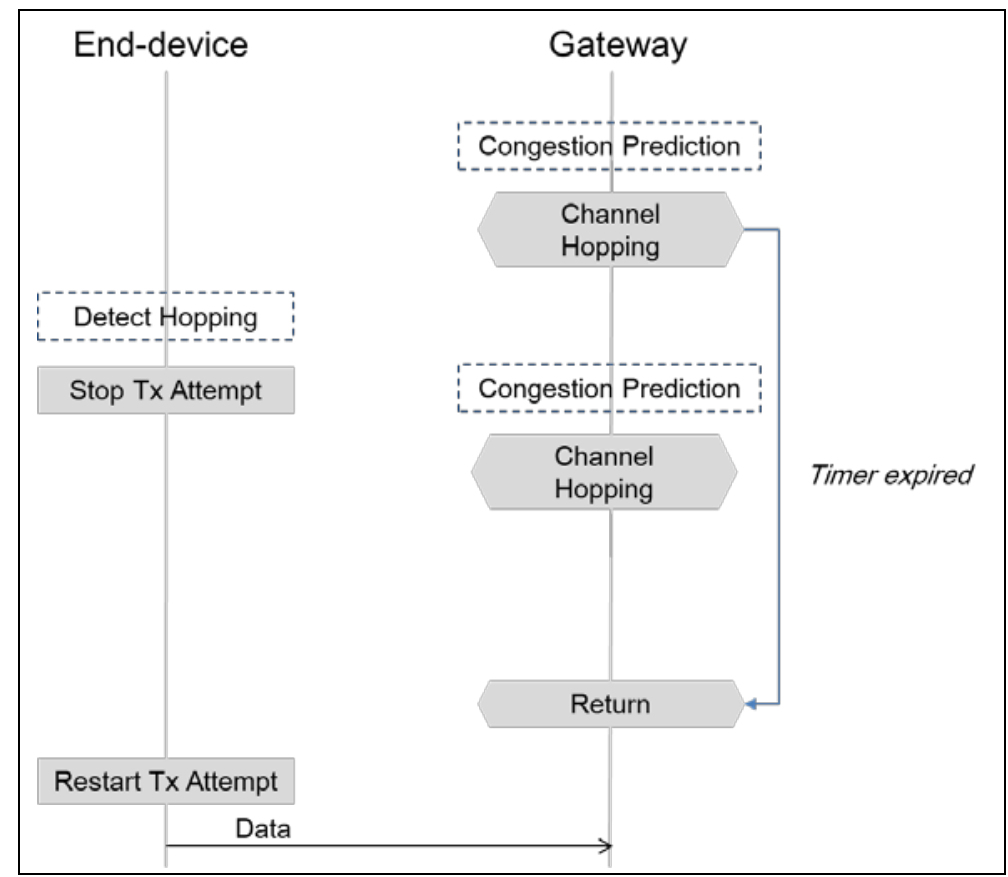

Fig. 4. The gateway’s operation when congestion occurs 


\section{Experimental Classification Results and Analysis}

The performance was evaluated using computer simulation, written in the Python language. The simulation time was 75,000 s and there was 20\% urgent traffic among the total generated traffic. The packet size was 200 bytes and was generated from a Poisson distribution with a lambda of 0.01. Congestion occurred according to the Poisson distribution with 0.01 lambda and was maintained for $500 \mathrm{~s}$. That is, congestion and non-congestion states were repeated over the wireless network during the simulation. The proposed approach performed channel hopping in the gateway if congestion occurred over the wireless network. The number of hopping channels was set to 12 and the data loss rate is assumed at 0.05 as a wireless link error. If congestion exists, it is assumed to be 0.35 , which is combined with the wireless link error and congestion error. If transmission failed, two retries were attempted.

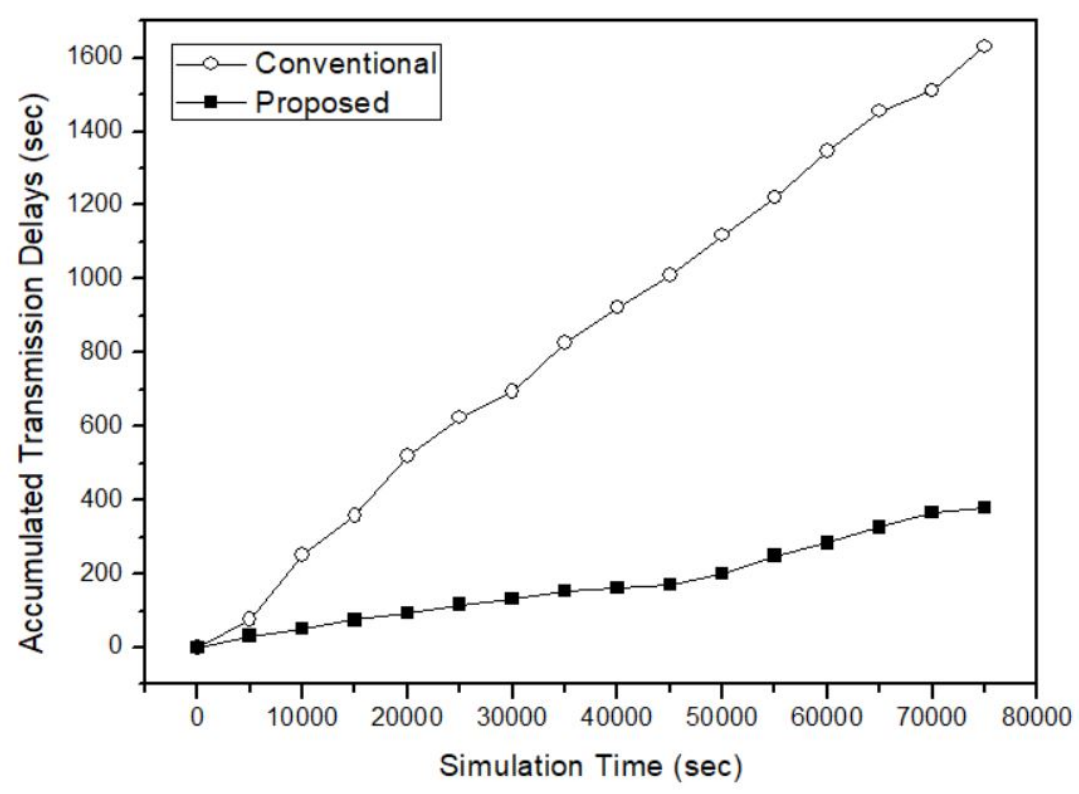

Fig. 5. Accumulated transmission delays

Fig. 5 shows accumulated delays during a simulation. The proposed scheme's accumulative delay is lower than that of the conventional scheme; if congestion occurs in LPWA networks, data traffic is concentrated on a gateway. These traffic loads reduce successful data transmissions and generate retransmissions to recover the lost data during data transmission; the retransmission causes long transmission delays. However, the proposed method changes the wireless channel to avoid the congested wireless channel. The proposed scheme can maintain transmission efficiency by avoiding congestion. That is, it reduces retransmission for data lost over wireless, which leads to improved transmission efficiency and reduced transmission energy consumption.

Fig. 6 shows the average data transmission rate during simulation. The proposed scheme transmits data traffic in a stable wireless environment by avoiding the congested wireless channel; it can maintain a higher transmission rate than conventional schemes. In general, LPWA technologies decrease the modulation level when transmission fails, which decreases the data transmission rate for the LPWA and retransmission. The proposed scheme can avoid 
transmission rate degradation by detecting congestion and moving the channel in advance. Therefore, the proposed scheme provides transmission efficiency in LPWA networks.

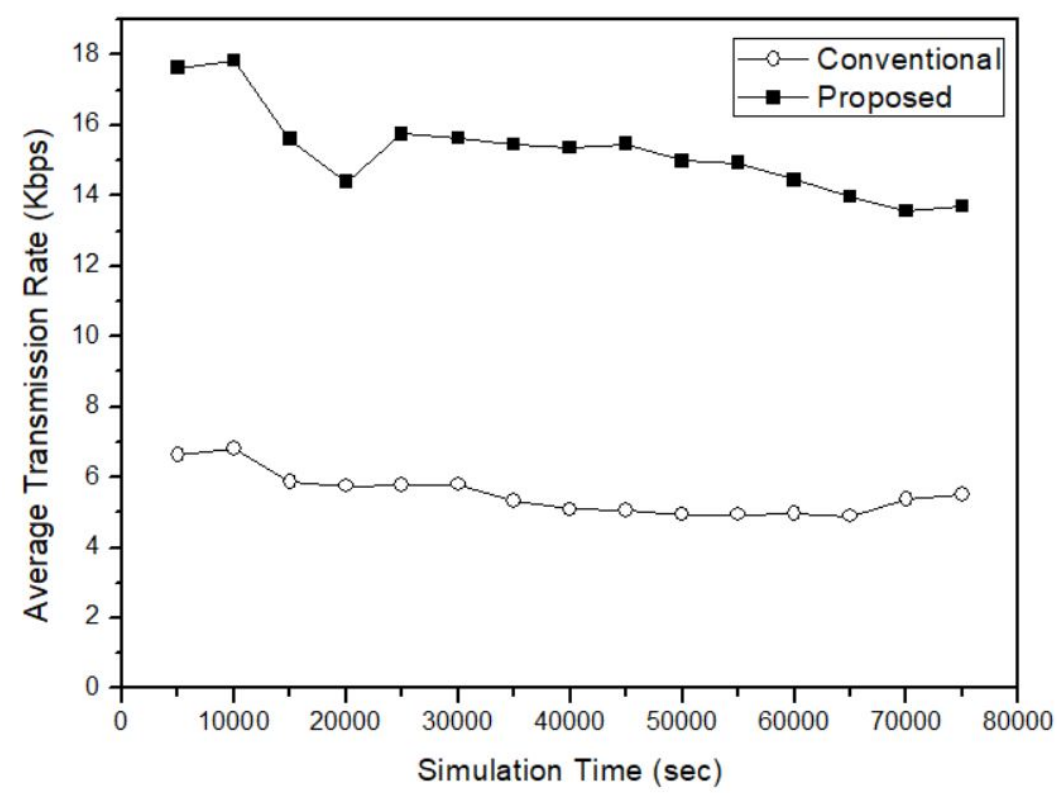

Fig. 6. Average transmission rate

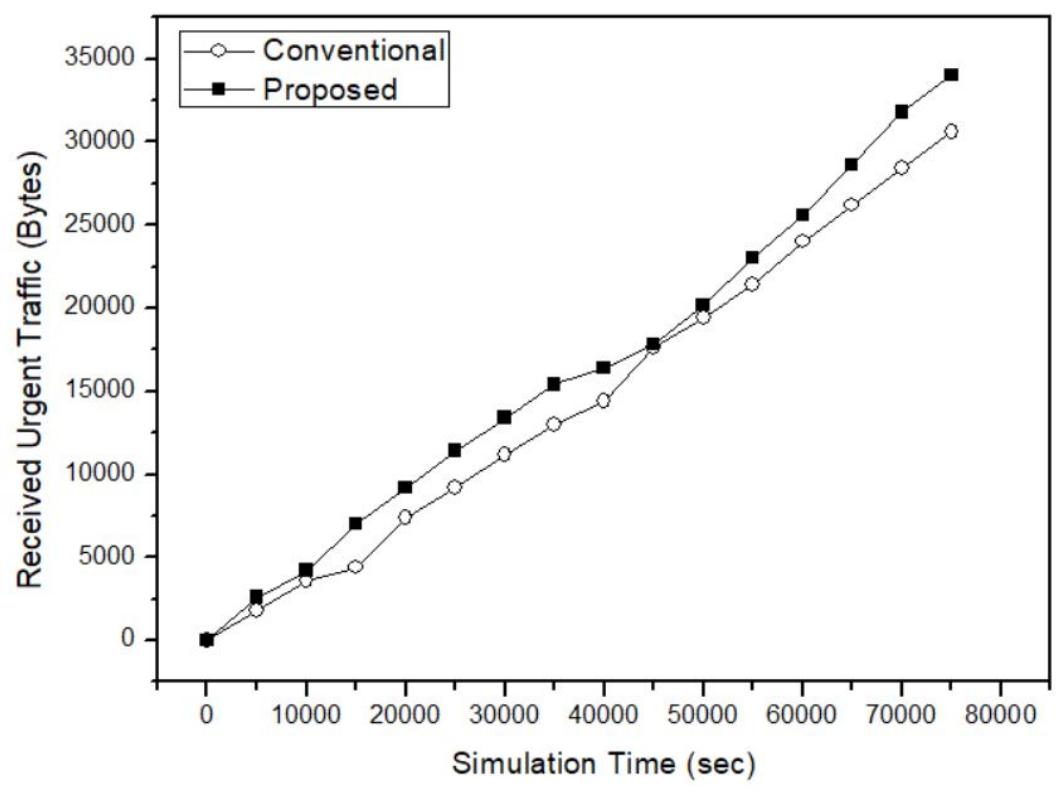

Fig. 7. Amount of urgent traffic received at a server

Fig. 7 represents amount of urgent data traffic transmitted successfully. As the figure shows, the proposed scheme successfully transmits a greater amount of urgent data because it avoids wireless congestion and thus reduces data lost in congestion; this can be also determined through Figures 5 and 6 . Avoiding congestion leads to lower transmission delays, a higher transmission rate, and LPWA networks have numerous neighbor devices because of 
the network's characteristics; therefore, wireless congestion is common. Congestion can greatly affect LPWA performance, so a scheme to increase transmission efficiency should be proposed even in congestion situations. The proposed scheme provides a novel approach to improving transmission efficiency in LPWA networks.

\section{Conclusion}

Long-range IoT services are composed of numerous end-devices that produce and transmit data to the Internet cloud via a gateway. Data traffic focuses at the gateway; thus, the gateway can frequently become congested. Congestion decreases the transmission efficiency in the wireless channel; avoiding congestion is important when providing efficient LPWA services. An LPWA network cannot avoid congestion by applying complex control mechanisms that use message exchanges because of long transmission delays. Although it allows for delays during data transmission, frequent message exchanges lead to substantial delays. In addition, its devices have insufficient resources. Data transmission should consider energy constraints; therefore, a simple congestion-avoidance mechanism is necessary.

This paper describes the gateway changing the wireless channel of LPWA to avoid congestion. If there are end-devices sending urgent data, they follow the gateway By changing their wireless channel to the gateway's changed channel. Other devices without urgent data stop sending and wait for the gateway to return. In the moved wireless channel, end-devices that followed the gateway can then transmit their urgent data. After a given time, the gateway will return to the original wireless channel, thus avoiding congestion in the original channel. Waiting end-devices (i.e., normal devices without urgent data) restart sending their data when the gateway returns; the proposed scheme avoids LPWA congestion and improves transmission efficiency.

\section{References}

[1] J. Gubbi, R. Buyya, S. Marusic, M. Palaniswami, "Internet of Things (IoT): A vision, architectural elements, and future directions,” Elsevier Future Generation Computer Systems, Vol. 29, No. 7, pp. 1645-1660, 2013. Article (CrossRef Link)

[2] W. Jing, Q. Miao, and G. Chen, “An open Scheduling Framework for QoS resource management in the Internet of Things,” KSII Transactions on Internet Information Systems, Vol. 12, No. 9, pp. 4103-4121, 2018. Article (CrossRef Link)

[3] D. Bandyopadhyay and J. Sen "Internet of Things: applications and challenges in technology and standardization,” Springer Wireless Personal Communications, Vol. 55, No. 1, pp. 49-69, 2011. Article (CrossRef Link)

[4] S.K. Kim, N. Sahu, and M. Preda, "Beginning of a New Standard: Internet of Media Things," KSII Transactions on Internet Information Systems, Vol. 11, No. 11, pp. 5182-5199, 2017.

Article (CrossRef Link)

[5] N. Sornin, M. Luis, T. Eirich, T. Kramp, and O. Hersent, "LoRaWAN specification," Lora Alliance LoRaWAN Specification, Release v1.0.2, 2016.

[6] A. Augustin, J. Yi, T. Clausen, and W.M. Townsley, "A study of LoRa: long range \& low power networks for the Internet of Things,” MDPI Sensors, Vol. 16, No. 9, ID1466, 2016.

Article (CrossRef Link)

X. Xiong, K. Zheng, R. Xu, W. Xiang, and P. Chatzimisios, "Low power wide area machine-to-machine networks: key techniques and prototype,” IEEE Communications Magazine, Vol. 53, No. 9, pp. 64-71, 2015. Article (CrossRef Link) 
[7] F. Adelantado, X. Vilajosana, P. Tuset-Peiro, B. Martinez, J. Melia-Segul, and T. Watteyne, "Understanding the limits of LoRaWAN," IEEE Communications Magazine, Vol. 55, No. 9, pp. 34-40, 2017. Article (CrossRef Link)

[8] U. Raza, P. Kulkarni, and M. Sooriyabandara, "Low power wide area networks: an overview," IEEE Communications Surveys \& Tutorials, Vol. 19, No. 2, pp. 855-873, 2017.

Article (CrossRef Link)

[9] M. Centenaro, L. Vangelista, A. Zanella, and M. Zorzi, "Long-range communications in unlicensed bands: the rising stars in the IoT and smart city scenarios," IEEE Wireless Communications, Vol. 23, No. 5, pp. 60-67, 2016. Article (CrossRef Link)

[10] LoRa Alliance. https://www.lora-alliance.org (2016). Accessed 15 Oct 2016.

[11] SIGFOX. http://www.sigfox.com (2016). Accessed 15 Oct 2016.

[12] Weightless. http://www.weightless.org (2016). Accessed 15 Oct 2016.

[13] D.Y. Kim and S. Kim, "Dual-channel medium access control of low power wide area networks considering traffic characteristics in IoE," Springer Cluster Computing - The Journal of Networks, Software Tools and Applications, Vol. 20, No. 3, pp. 2375-2384, 2017. Article (CrossRef Link)

[14] D.Y. Kim, S. Kim, H. Hassan, and J.H. Park, "Adaptive data rate control in low power wide area networks for long range IoT services," ELSEVIER Journal of Computational Science, Vol. 22, pp. 171-178, 2017. Article (CrossRef Link)

[15] D.Y. Kim, S. Kim, H. Hassan, and J.H. Park, "Radio resource management for data transmission in low power wide area networks integrated with large scale cyber physical systems," Springer Cluster Computing - The Journal of Networks, Software Tools and Applications, Vol.20, No.2, pp.1831-1842, 2017. Article (CrossRef Link)

[16] I. Israr, M.M. Yaqoob, N. Javaid, U. Qasim, and Z.A. Khan, "Simulation analysis of medium access techniques," In Proc. of IEEE International Conference on Broadband, Wireless Computing, Communication and Applications, pp. 602-607, 2012. Article (CrossRef Link)

[17] S. Ahmed and S.S. Kanhere, "A bayesian routing framework for delay tolerant networks," in Proc. of IEEE wireless communications and networking conference (WCNC), 2010.

Article (CrossRef Link)

[18] S. Marsland, Machine learning an algorithmic perspective, Chapman \& Hall, New York, USA, 2009.

[19] D.Y. Kim, D. Ko, and S. Kim, "Network access control for location-based mobile services in heterogeneous wireless networks,” Mobile Information Systems, Vol. 2017, ID 6195024, 2017. Article (CrossRef Link)

[20] M.A. Alsheikh, S. Lin, D. Niyato, and H.-P. Tan, "Machine learning in wireless sensor networks: algorithms, strategies, and applications,” IEEE Communications Surveys \& Tutorials, Vol. 16, No. 4, pp. 1996-2018, Apr. 2014. Article (CrossRef Link)

[21] Z. Jin, Y. Han, J. Cho, and B. Lee, "A prediction algorithm for coexistence problem in multiple-WBAN environment,” International Journal of Distributed Sensor Networks, Vol. 2015, ID 386842, 2015. Article (CrossRef Link)

[22] Z. Lin, F. Xiao, Y. Sun, Y. Ma, C.C. Xing, and J. Huang, “A Secure Encryption-Based Malware Detection System,” KSII Transactions on Internet Information Systems, Vol. 12, No. 4, pp. 1799-1818, 2018. Article (CrossRef Link) 


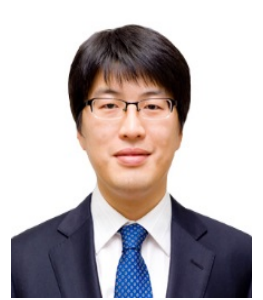

Dae-Young Kim received his B.E. degree in Electronics Engineering, M.S. and Ph.D. degrees in Computer Engineering from Kyung Hee University, Korea in 2004, 2006, and 2010, respectively. From 2010 to 2013, he was a Research Staff in the Communication R\&D Lab. at LIG Nex1 Co., Ltd., Korea. From 2013 to 2015, he was a Research Staff at AirPlug, Inc., Korea. From 2015 to 2017, he was an Assistant Professor in the Department of Software Engineering at Changshin University, Korea. Currently, he is an Assistant Professor in the School of Information Technology Engineering at Daegu Catholic University, Korea. His research interests include Mobile Networking \& Computing, IoT Networking and Machine Learning for Network Systems.

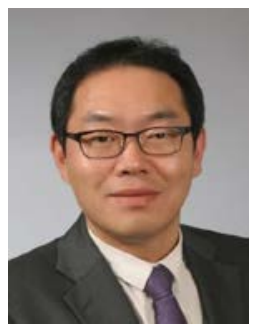

Seokhoon Kim received the B.E. and Ph.D. degrees in computer engineering from Kyunghee University, Korea, in 2000 and 2004, respectively. From 2004 to 2006, he was with IPOne, Inc., Seoul, Korea, where he led various research projects as a Research Engineer. From 2006 to 2009, he was a Research Engineer at Neowave, Inc., Anyang, Korea, where he developed Mobile WiMAX (IEEE 802.16) devices. He was an Assistant Professor in the Department of Mobile Communications Engineering at Changshin University, Changwon, Korea. Since March 2016, he has been with the Department of Computer Software Engineering, Soonchunhyang University, Asan, Korea, where he is currently an Assistant Professor. His research interests comprise Cloud Computing, Internet of Things, Software Defined Networking, Mobile System/Communications, and Machine Learning based on Bigdata. 